\title{
Bilateral Optical Power Meter Comparison Between NIST and CENAM*
}

Number 4

July-August 2008

\author{
Volume 113 \\ I. Vayshenker, X. Li, \\ D. J. Livigni, and J. H. Lehman \\ Optoelectronics Division, \\ National Institute of Standards \\ and Technology, \\ Boulder, CO 80305

\section{J. C. Bermudez, J. C. Molina, Z. E. Ruiz} \\ Centro Nacional de Metrología, \\ km 4,5 carretera a los Cués, \\ Municipio El Marqués, \\ C.P. 76241 , \\ Queretaro, México \\ igor.vayshenker@nist.gov \\ xiaoyu.1i@nist.gov \\ dlivigni@nist.gov \\ jlehman@nist.gov \\ jbermude@cenam.mx \\ jmolina@cenam.mx \\ zruiz@cenam.mx
}

\author{
We describe the results of a comparison of \\ reference standards between the National \\ Institute of Standards and Technology \\ (NIST-USA) and Centro Nacional \\ De Metrología (CENAM-Mexico). Open \\ beam (free field) and optical-fiber-based \\ measurements at wavelengths of \\ $1302 \mathrm{~nm}$ and $1546 \mathrm{~nm}$ are reported. Both \\ laboratories' reference standards were \\ compared by means of a temperature- \\ controlled optical trap detector. \\ Measurements showed a largest difference \\ of less than 3.4 parts in $10^{3}$, which is \\ within the combined expanded $(k=2)$ \\ uncertainty for the laboratories' reference \\ standards.
}

Key words: international comparison; optical fiber; optical power.

Accepted: July 9, 2008

\section{Introduction}

In our previous work [1-5], we reported the results of international comparisons of reference standards used in the calibration of optical power meters. Those reports describe the results that were obtained by use of open laser beams $[1,5]$ and optical fiber cable [2-5] at wavelengths of 1302 and $1546 \mathrm{~nm}$. We also compared internal NIST laser and optical fiber power reference standards at several laser wavelengths in the visible and near infrared (NIR) [6]. In this paper, we compare the reference standards maintained by CENAM and NIST laboratories by launching optical power from an open beam and an optical fiber.
For optical fiber power meter measurements, the primary standard for each laboratory is a cryogenic radiometer described in [7,8]. Most primary standards are designed to be used with collimated (open) beams rather than divergent beams from an optical fiber. Reference standards are typically calibrated against the primary standards by means of collimated beams, but are used with divergent beams characteristic of laser light exiting an optical fiber. A transfer standard that is insensitive to beam geometries (either collimated or divergent beam) is a very important tool for comparing reference standards.

\footnotetext{
* Partial contribution of the National Institute of Standards and Technology; not subject to copyright
} 


\section{Transfer Standard}

For this comparison we used a transfer standard designed and built by NIST [9]. The transfer standard depicted in Fig. 1 is an optical-trap detector that consists of two germanium photodiodes and a spherical mirror. The trap detector has two Ge photodiodes $10 \mathrm{~mm}$ in diameter and a concave aluminum mirror of $15 \mathrm{~mm}$ diameter and $40 \mathrm{~mm}$ focal length. The mirror is coated with magnesium fluoride. The two photodiodes are oriented relative to the entrance aperture so that the principal ray of incident radiation strikes each diode once at a $45^{\circ}$ angle of incidence and then reflects from the concave mirror back onto the photodiodes in reverse order. The photodiodes and mirror are enclosed in a thermoelectrically cooled environment. It has been shown in [10] that such a configuration provides a uniform response over the field of view and therefore requires no correction for beam geometry. A Ge-trap detector was calibrated at both national laboratories against their reference standards. The same lasers, operating at wavelengths of $1302 \mathrm{~nm}$ and $1546 \mathrm{~nm}$, and same optical fiber cable with fiber connectors with

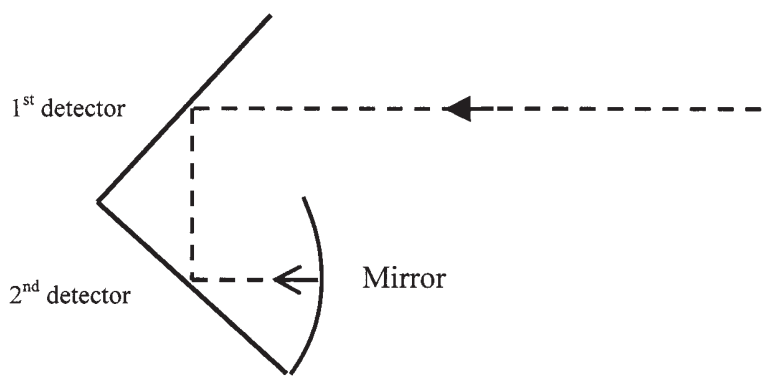

Fig. 1. Germanium-trap detector.

physical contact (FC/PC) were used by both laboratories, which employed a direct substitution method for their measurements.

\section{Measurement System}

The NIST and CENAM measurement systems are very similar; therefore in this section we describe the common measurement system used by both laboratories. The measurement system is depicted in Fig. 2; it

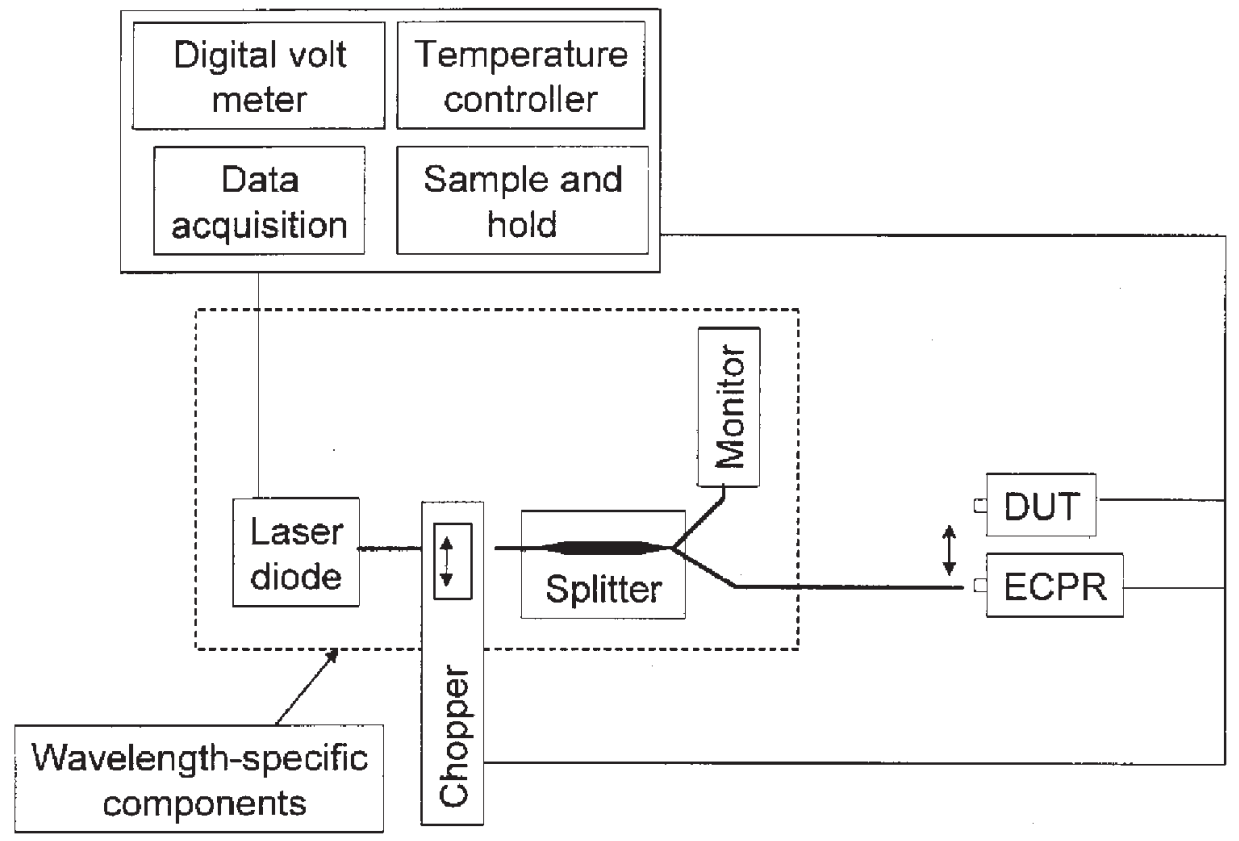

Fig. 2. Measurement system that utilizes fiber-pigtailed laser diode sources at wavelengths of 1302 and $1546 \mathrm{~nm}$. 
consists of fiber-pigtailed laser diode sources at wavelengths of $1302 \mathrm{~nm}$ and $1546 \mathrm{~nm}$, a reference optical fiber cable, and a positioning stage (see double-headed arrow) for comparing the reference and transfer (DUT) standards. The NIST measurement system is described in more detail in [11]. Both laboratories' reference standards are electrically calibrated pyroelectric radiometers (ECPRs) that have been previously calibrated against the primary standards described in $[7,8]$. The ECPR consists of a thermal detector that is covered with a gold black coating. The response of the ECPR does not depend on the wavelength of the incident radiation over the wavelength region of $1300 \mathrm{~nm}$ to $1550 \mathrm{~nm}$ [12]. The power was approximately $100 \mu \mathrm{W}$, or $-10 \mathrm{dBm}$ for both optical fiber/connector and open beam configurations.

\section{Results of the Comparison}

\subsection{Using Optical Fiber Cable}

Both participating laboratories used the same optical fiber cable with FC/PC connectors. At NIST, six measurement runs were performed with relative standard deviations of $1.2 \times 10^{-3}$ at both wavelengths of $1302 \mathrm{~nm}$ and $1546 \mathrm{~nm}$. At CENAM, nine measurement runs were performed with a relative standard deviation of $4 \times 10^{-4}$ at $1302 \mathrm{~nm}$ and a relative standard deviation of $1 \times 10^{-3}$ at $1546 \mathrm{~nm}$. The results of the comparison are presented in Table 1 .

Table 1. Results of NIST and CENAM comparison using the optical fiber cable

\begin{tabular}{ccc}
\hline \hline $\begin{array}{c}\text { Source wavelength } \\
(\mathrm{nm})\end{array}$ & $\begin{array}{c}\text { difference } \\
(\%)\end{array}$ & $\begin{array}{c}\text { combined expanded }(k=2) \\
\text { uncertainty }(\%)\end{array}$ \\
\hline 1302 & 0.20 & 0.48 \\
1546 & -0.03 & 0.56 \\
\hline
\end{tabular}

The standard uncertainties for the optical power measurements were evaluated in accordance with ISO document standards [13]. At $1302 \mathrm{~nm}$, the difference between the NIST and CENAM results was 2 parts in $10^{3}$, and at $1546 \mathrm{~nm}$ the difference was 3 parts in $10^{4}$ (minus sign for the difference indicates that the Ge trap detector responsivity measured by CENAM is lower than that measured by NIST). The NIST combined standard uncertainty was 2 parts in $10^{3}$ at $1302 \mathrm{~nm}$ and 2.5 parts in $10^{3}$ at $1546 \mathrm{~nm}$, while that of CENAM's was 1.4 parts in $10^{3}$ at both wavelengths of $1302 \mathrm{~nm}$ and $1546 \mathrm{~nm}$. Table 1 provides values of relative combined standard uncertainty for both laboratories. These values were calculated by taking a square root of the sum of the squares of each laboratory's combined uncertainty. A more detailed uncertainty analysis can be found in [11]. The observed interlaboratory differences are less than the combined standard $(k=1)$ uncertainties for the laboratories' reference standards.

\section{2 Using Open Beam}

Each participating laboratory used the same beam size at both wavelengths. At NIST, six measurement runs were performed with a relative standard deviation of $0.7 \times 10^{-3}$ at a wavelength of $1302 \mathrm{~nm}$ and a relative standard deviation of $1.5 \times 10^{-3}$ at a wavelength of $1546 \mathrm{~nm}$. At CENAM, nine measurement runs were performed with a relative standard deviation of $6 \times 10^{-4}$ at $1302 \mathrm{~nm}$ and a relative standard deviation of $5 \times 10^{-4}$ at $1546 \mathrm{~nm}$. The beam size at both wavelengths was $1.7 \mathrm{~mm} \pm 0.1 \mathrm{~mm}$ in diameter at the $1 / \mathrm{e}^{2}$ intensity points. The results of the comparison are presented in Table 2.

Table 2. Results of NIST and CENAM comparison using using open beam

\begin{tabular}{ccc}
\hline \hline $\begin{array}{c}\text { Source wavelength } \\
(\mathrm{nm})\end{array}$ & $\begin{array}{c}\text { difference } \\
(\%)\end{array}$ & $\begin{array}{c}\text { combined expanded }(k=2) \\
\text { uncertainty }(\%)\end{array}$ \\
\hline 1302 & -0.14 & 0.38 \\
1546 & -0.34 & 0.50 \\
\hline
\end{tabular}

At $1302 \mathrm{~nm}$, the difference between the NIST and CENAM results was 1.4 parts in $10^{3}$, and at $1546 \mathrm{~nm}$ the difference was 3.4 parts in $10^{3}$ (minus sign for the difference indicates that the Ge trap detector responsivity measured by CENAM is lower than that measured by NIST). The NIST combined standard uncertainty $(k=1)$ was 1.7 parts in $10^{3}$ at $1302 \mathrm{~nm}$ and 2.3 parts in $10^{3}$ at $1546 \mathrm{~nm}$, while that of CENAM's was 9 parts in $10^{4}$ at $1302 \mathrm{~nm}$ and 1 part in $10^{3}$ at $1546 \mathrm{~nm}$. Table 2 provides values of relative combined expanded uncertainties for NIST and CENAM. These values are calculated by taking a square root of the sum of the squares of each laboratory combined uncertainty. The observed interlaboratory differences are less than the relative combined expanded $(k=2)$ uncertainties for the laboratories' reference standards. 


\section{Conclusion}

This optical power meter comparison shows reasonably good agreement within the combined expanded uncertainty between NIST and CENAM. The agreement between these two laboratories is in harmony with the previous international comparisons described in [1-5]. Such comparisons are important to establish a worldwide consistency in measurements of optical power for optical telecommunications.

\section{References}

[1] I. Vayshenker, H. Haars, X. Li, J. H. Lehman, and D. J. Livigni, Comparison of optical-power meters between NIST and PTB, Metrologia 37, 349-350 (2000).

[2] S. V. Tikhomirov, A. I. Glazov, M. L. Kozatchenko, V. E. Kravtsov, A. B. Svetlichny, I. Vayshenker, T. R. Scott, and D. L. Franzen, Comparison of reference standards for measurements of optical-fibre power, Metrologia 37, 347-348, (2000).

[3] I. Vayshenker, H. Haars, X. Li, J. H. Lehman, and D. J. Livigni, Optical fiber-power meter comparison between NIST and PTB, J. Res. Natl. Inst. Stand. Technol. 108, 391-394 (2003).

[4] I. Vayshenker, D. J. Livigni, J. A. Hadler, and J. H. Lehman, NIST optical fiber power measurements: intramural and international comparisons, Digest, Conf. Optical Fibre Measurement Conference, 97-100, Teddington (2007).

[5] I. Vayshenker, J. H. Lehman, D. J. Livigni, X. Li, K. Amemiya, D. Fukuda, S. Mukai, S. Kimura, M. Endo, J. Morel, and A. Gambon, Trilateral optical power meter comparison between NIST, NMIJ/AIST, and METAS, Appl. Opt. 46 (5), 643-647 (2007).

[6] J. H. Lehman, I. Vayshenker, D. J. Livigni, and J. A. Hadler, Intramural comparison of NIST laser and optical fiber power calibrations, J. Res. Natl. Inst. Stand. Technol. 109, 291-298 (2004).

[7] D. J. Livigni, High accuracy laser power and energy meter calibration service, NIST Special Publication 250-62 (2003).

[8] K. D. Stock, H. Hofer, J. G. Suarez Romero, L. P. Gonzalez Galvan, and W. Schmid, Cryogenic radiometer facility of the CENAM and first international comparison, Metrologia 37, 269-271 (2000).

[9] J. H. Lehman and C. L. Cromer, Optical trap detector for calibration of optical fiber powermeters: coupling efficiency, Appl. Opt. 31, 6531-6536 (2002).

[10] J. H. Lehman and X. Li, A transfer standard for optical fiber power metrology, Eng. and Lab. Notes in Opt. \& Phot. News, Vol. 10 (5), May 1999, archived in Appl. Opt. 38 (34), 71647166 (1999).

[11] I. Vayshenker, X. Li, D. J. Livigni, T. R. Scott, and C. L. Cromer, Optical fiber power meter calibrations at NIST, NIST Special Publication 250-54 (2000).

[12] C. A. Hamilton, G. W. Day, and R. J. Phelan Jr., An electrically calibrated pyroelectric radiometer system, Nat. Bur. Stand. (U.S.) Tech. Note 678, March 1976.

[13] ISO, Guide to the Expression of Uncertainty in Measurement, International Organization for Standardization, Geneva, Switzerland (1993).
About the authors: Igor Vayshenker, David Livigni, Xiaoyu Li are electronics engineers and calibration leaders. John H. Lehman is a physicist and project leader for Laser Radiometry in the Optoelectronics Division of the NIST Electronics and Electrical Engineering Laboratory. J. C. Bermudez is a leader of Detectors Group, J. C. Molina and Z. E. Ruiz are calibration engineers in the Optics and Radiometry Division of CENAM. The National Institute of Standards and Technology is an agency of the U.S. Department of Commerce. CENAM is the national metrology institute of México. 